\title{
Estrés Laboral Y Afectación Psico-Física En El Rendimiento Del Personal En Un Call Center Policial, Quito-Ecuador
}

\author{
MD. Pedro Flores Brito \\ Magister en Seguridad y Prevención de Riesgos del Trabajo \\ Docente Escuela Superior Politécnica de Chimborazo, Ecuador \\ MD. Shirley Fernanda Rosero \\ Magister en Gerencia en Salud para el Desarrollo Local \\ Docente de Escuela Superior Politécnica de Chimborazo, Ecuador
}

MD. Daniel Renato Reinoso

Especialista en Anestesiología

Docente en la Facultad de Medicina - ESPOCH, Ecuador

Doi: 10.19044/esj.2018.v14n24p88～URL:http://dx.doi.org/10.19044/esj.2018.v14n24p88

\begin{abstract}
The study investigates the various aspects of the perception of the health of call-center workers. This paper focuses on identifying the psychophysical affectation in the work performance of workers due to work stress from the foundation of preventive health. This, however, associated the relationship between the two variables to verify its affectation. Occupational stress influences the occurrence of psycho-physical alterations that affect work performance. This is attributed to the fact that it was found in the group investigated, which is composed of workers of the police operational management center of the metropolitan district of Quito (CGOP). The Maslach universal test that measures Burnout syndrome, emotional fatigue, depersonalization, and personal fulfillment were combined with attitude tests with Likert scales that measure the perception of those involved with their psychophysical affectation at their place of work. The results are evidenced, according to the Maslach test, in relation to the suffering of Burnout syndrome. The result shows that in this study, no worker meets the three conditions: emotional exhaustion (10.5\%), depersonalization (22.2\%), or realization personal $(22.3 \%)$. However, they do meet one of the three areas individually. Associated with stress, physical-psychological affectations were identified, especially in the nervous system (1 in 10) and to a lesser extent in the digestive system. In conclusion, the results are mostly isolated because the study group performs frequent physical activity, which considerably diminishes the secondary and negative effects on their physical health.
\end{abstract}


Keywords: Work stress, psycho-physical affectation, personal performance, Burnout syndrome

\section{Resumen}

La presente investigación considera los aspectos sobre la percepción de la salud que tienen los trabajadores de un call-center. El estudio se enfoca en identificar la afectación psico-física en el rendimiento laboral a causa del estrés laboral, desde la fundamentación de la salud preventiva, asociando la relación entre las dos variables para comprobar su afectación. El estrés laboral influye en el aparecimiento de las alteraciones psico-físicas que afectan en el rendimiento laboral, puesto que se ha podido comprobar en el grupo investigado que lo componen los trabajadores de la central de gestión operativa policial del distrito metropolitano de Quito (CGOP), usando el test universal de Maslach que mide síndrome Burnout, cansancio emocional, despersonalización, y realización personal, se combinan con test de actitud con escalas Likert que miden percepción de los involucrados en torno a su afectación psicofísica en su lugar de trabajo. Los resultados se evidencian de acuerdo al test de Maslach, en relación al padecimiento del síndrome de Burnout que, en el estudio ningún trabajador cumple con las tres condiciones: agotamiento emocional $(10,5 \%)$, despersonalización $(22,2 \%)$ o realización personal $(22,3 \%)$, sin embargo, sí cumplen en alguna de las tres áreas de manera individual. De manera asociada al estrés se identificaron afectaciones físico-psicológicas, especialmente en el sistema nervioso (1 de cada 10) y en menor proporción en el sistema digestivo. Concluyendo que los resultados son mayoritarios porcentualmente aislados debido a que el grupo de estudio realiza frecuente actividad física, lo que disminuye considerablemente los efectos secundarios y negativos en su salud física.

Palabras Clave: Estrés laboral, afectación psico-física, rendimiento personal, síndrome de Burnout

\section{Introducción}

La concepción de salud, describe un completo estado de bienestar biopsico, social y no solo la ausencia de enfermedad, esta descripción hace ver la necesidad de analizar de manera integral la salud de la población. En esta corriente, se permite entender el entorno laboral, como el espacio en donde el hombre desarrolla y permanece una gran parte del tiempo, lo cual conlleva a pensar las implicaciones que tiene el servicio laboral en la vida integral del ser humano (Restrepo \& Málaga, 2011).

La naturaleza laboral ha experimentado una transformación importante en las últimas décadas en el contexto sociocultural, la internacionalización 
económica y la innovación tecnológica, esto ha generado un proceso de cambios significativos, primero porque se han ido reduciendo las demandas físicas y segundo, se han ido incrementando las demandas sobre la interacción psicosocial (Segurado Torres \& Agulló Tomás, 2004).

El problema del estrés asociado a las afectaciones físicas y psicológicas que desarrollan los trabajadores en países en vías de desarrollo, es un fenómeno observable por la frecuente recurrencia de los casos que se incrementan directamente proporcional al avance de las tecnologías y al desarrollo de las automatizaciones, que imposibilitan las actividades psicosociales propias del ser humano en todo ámbito (Jiménez \& León, 2010).

Si se relaciona el estrés, las condiciones de trabajo, las expectativas de los trabajadores y el rendimiento laboral, se puede dimensionar que un fenómeno es interdependiente de otro, lo cual, prescribe que toda intervención ocupacional deberá ser asumida en el contexto integral, y no como elementos aislados, por tanto, el estrés es el resultado de la aglomeración de situaciones que el trabajador las asume con el pretexto de la responsabilidad y la angustiosa necesidad de mantenerse en su sitio de trabajo, lo cual, deja entrever que las condiciones laborales, aunque se han mejorado en parte, siguen siendo aterradoramente la causa de las lesiones físicas y psicológicas que sufren a diario los trabajadores y trabajadoras.

El rendimiento laboral, por su parte, se lo concibe como el valor total que la empresa espera con respecto a lo que un trabajador lleva a cabo en un período de tiempo determinado, ese valor, que puede ser positivo o negativo, en función de que el empleado presente un buen o mal rendimiento, supone la contribución que ese empleado hace a la consecución de la eficacia de su organiza (Motowidlo, 2004).

Se aprecia, la creciente problemática en todos los extractos laborales, tanto superiores como inferiores, de ahí que el estrés laboral no persigue un grupo específico de trabajadores, por tanto, la importancia de identificar a tiempo algunas alteraciones que pueden derivarse del aparecimiento del estrés por el trabajo, lo cual se muestra en las afectaciones de orden psicológico y físico (Silla, 2011).

La Organización Mundial de la Salud catalogo al estrés como una epidemia Mundial y exhorto a trabajar en este problema que se ha vuelto global, ya que considera que el estrés va a afectar negativamente a la salud psicológica y física de los trabajadores, lo que a su vez tiene un efecto directo sobre la eficiencia y productividad que puedan brindar estas personas a sus respectivas empresas; entendiendo productividad como la capacidad de producción personal de acuerdo a los recursos disponibles con la mayor eficiencia posible (Moriana Elvira \& Herruzco, 2005).

Este fenómeno se puede observar claramente en los servidores estatales que atienden llamadas diariamente y tienen que lidiar con diferentes 
situaciones, actitudes, problemas, reclamos, urgencias, obligaciones que van de a poco, si no son manejadas adecuadamente, provocando alteraciones psicológicas, especialmente estrés, que puede terminar afectando en su capacidad de respuesta y productividad.

Este precisamente es el caso de los trabajadores del Call Center de la Central de Gestión Operativa Policial del Distrito Metropolitano de Quito (CGOP), ellos tienen la labor durante toda su jornada profesional el atender llamadas telefónicas para darles información y soluciones a diversos tipos de problemas o inquietudes, incluida la atención y respuesta policial según sea el caso o la derivación hacia otros servicios urgentes, entre otras, lo que podría ocasionar un alto grado de estrés o incluso el aparecimiento del síndrome de burnout, lo que a su vez repercutiría sobre su rendimiento y capacidad de resolución de problemas. Es importante entonces determinar, ¿Cuál es el efecto directo que el aparecimiento del estrés laboral tiene sobre la capacidad de trabajo o rendimiento laboral de los trabajadores del call center?

\section{Objetivo de la investigación}

Comprobar la presencia de estrés laboral, desde la fundamentación científica de la salud preventiva en los trabajadores de la Central de Gestión Operativa Policial del Distrito Metropolitano de Quito.

Identificar las afectaciones psico-físicas en el rendimiento laboral de los trabajadores del área del call center de la Central de Gestión Operativa Policial del Distrito Metropolitano de Quito.

\section{Metodología}

La metodología general está dada por la realización de diferentes tipos de test tipo encuestas, que fueron respondidas personalmente por los trabajadores del call center, luego se procedió a realizar el respectivo análisis e interpretación de los resultados.

Dentro de la metodología para el diagnóstico del estrés se define desde la planificación de indicadores: cansancio emocional, despersonalización, y realización personal, para lo cual se usó como herramienta de recolección y medición de indicadores el test de Maslach para determinar el síndrome de Burnout en el grupo de estudio.

Para estudiar las alteraciones psico - físicas, primero se elaboró el diseño de una herramienta de recolección de información, tipo encuesta, la misma que se basa en la escala de valoración tipo Likert.

Para hacer referencia al rendimiento laboral, se determina la construcción de un componente adicional al banco de preguntas que componen la encuesta general. 


\section{Técnicas para la obtención de datos}

Entre las técnicas principales para la obtención de datos, se encuentran la aplicación de instrumentos científicamente probados y demostrados en el diagnóstico del síndrome de Burnout que es el test de Maslach, acompañada de una encuesta trabajada especialmente para recoger información que responda a los indicadores propuestos (previamente validados con un grupo control), estos dos instrumentos se aplican y sirven para el levantamiento y la recolección de datos primarios de la investigación.

Se trabaja además con la técnica bibliográfica para la compilación de información teórica y científica útil para la comprensión y análisis de los contenidos, así como para la descripción de los hallazgos, estas fuentes, consideradas secundarias, serán el marco de soporte para la investigación.

\section{Cálculo de la Muestra}

Para el cálculo de la muestra, se parte de un universo que es el número total de trabajadores del call center de la Central de Gestión Operativa Policial del Distrito Metropolitano de Quito, a partir del segundo semestre del año 2016 y primero del 2017, que es de 60 trabajadores. Se realiza el cálculo de la muestra en una población menor de 100 personas por el motivo del difícil acceso hacia la población total, la Institución Policial maneja políticas internas que no permiten, interactuar adecuadamente con el total de los trabajadores. Para el cálculo de la muestra para el estudio, se realizó en función de la fórmula estadística de muestreo probabilístico al 95\% de confiabilidad, que se muestra a continuación:

Fórmula. Muestreo Probabilístico

$$
n=\frac{Z^{2} p q N}{N e^{2}+Z^{2} p q}
$$

Dónde:

$$
\begin{array}{ll}
\mathrm{n}=\text { Tamaño de la muestra } & =i ? \\
\mathrm{Z}=\text { Nivel de confianza } 95 \% & =1.69 \\
\mathrm{p}=\text { Probabilidad a favor } & =0.50 \\
\mathrm{q}=\text { Probabilidad en contra } & =0.50 \\
\mathrm{~N}=\text { Población total } & =60 \\
\mathrm{e}=\text { Error de estimación } & =0.05
\end{array}
$$

Luego de reemplazar los datos en la fórmula, el tamaño de la muestra es igual a 38 trabajadores. La selección de la muestra es aleatoria y se basa en los parámetros anotados en la descripción del proceso, puesto que los 
trabajadores son distribuidos en 4 grupos diferentes, cada uno de estos grupos labora durante 8 horas ininterrumpidas, y no existe la posibilidad de tener acceso para el personal civil, pues éste es restringido y de uso exclusivo para personal policial. Con estas condiciones laborales y de acceso, se justifica que la selección sea aleatoria.

\section{Resultados y Discusión}

\section{Datos Generales de la Población de Estudio}

Distribución por edad y sexo

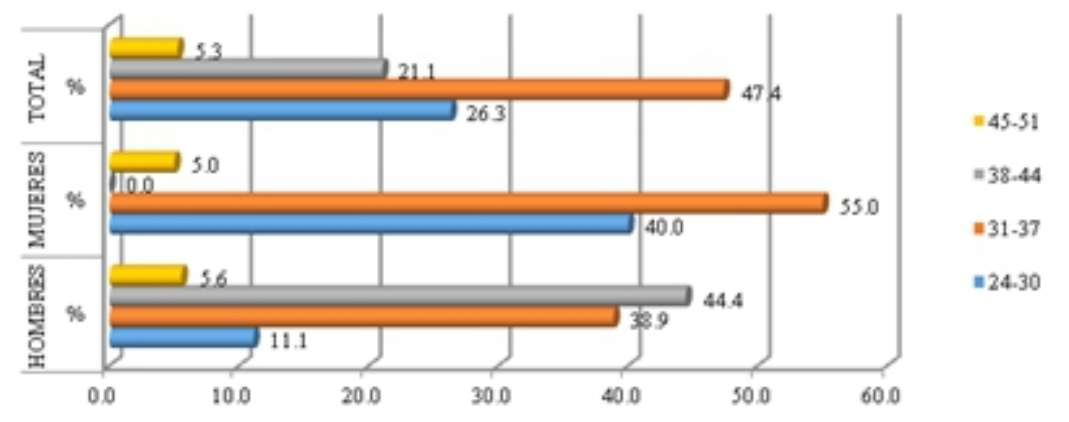

Gráfico 1. Distribución según edad y sexo de los Trabajadores

Fuente: Investigación primaria, 2018.

Dentro de la Central de Gestión Operativa Policial del Distrito Metropolitano de Quito, operan 4 grupos de policías de diferentes rangos de jerarquía, y de ambos sexos, se trata de dinamizar la comprensión de los resultados al igualar los dos géneros hacia el cien por ciento para cada una de ellas, y se distribuyen en grupos de edad con un rango de 7 para representar de manera equilibrada (Roldán, 2004). Nótese que los rangos de edad se encuentran entre 24 y 51 años de edad, el promedio de edad de los trabajadores de ambos sexos es de 34,7 años, variando significativamente si se comparan los géneros de manera individual, donde el promedio de edad de hombres es de 37,2 años, y de mujeres es de 32,4 años.

\section{Porcentaje de trabajadores por sexo y sus cargas familiares}

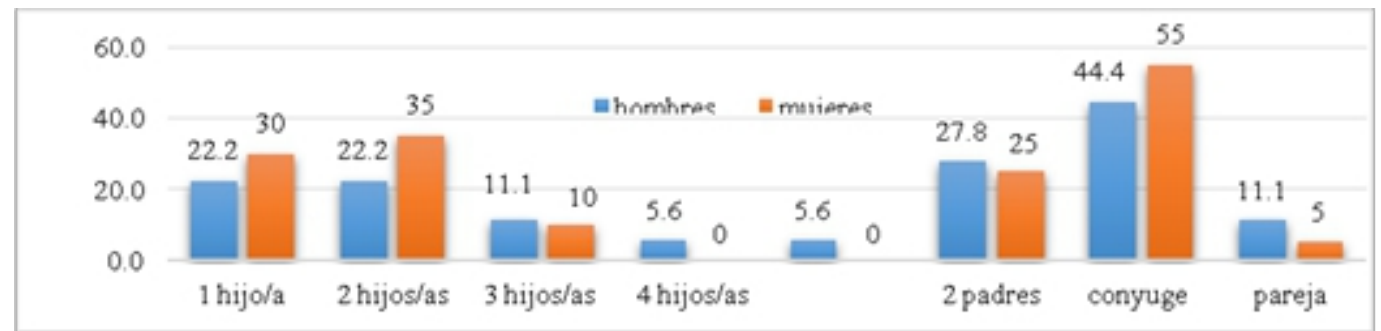

Gráfico 2. Cargas familiares de los trabajadores del call center

Fuente: Investigación primaria, 2018. 
Sin duda, una de las primeras causas de preocupación es la fuente de ingreso, especialmente cuando el trabajador tiene la responsabilidad de una familia en sus hombros por tanto, el hallazgo evidencia esta condición en el grupo investigado, dado que, en su rol de padre, madre, hijo, hija, esposo o relación de pareja, el entenderlos como carga familiar, constituye un factor determinante que puede generar estrés (Houtman, Jettinghoff, \& Cedillo, 2008). Este análisis recoge una realidad inclinada hacia el rol de la mujer en la sociedad, en la gráfica se demuestra que la mayor carga familiar la tienen las mujeres, notándose significativamente en el número de hijos e hijas de 1 a 2 con diferencias porcentuales de 8 y $13 \%$ respectivamente, y en la responsabilidad que tienen con sus padres donde existe una pequeña diferencia entre sexos, de 2,8\% del hombre comparada a la mujer. El fenómeno vuelve a presentarse en la relación conyugal, donde la diferencia es del $11 \%$ de responsabilidad de las mujeres sobre el resultado de los hombres. Nótese un dato interesante en cuanto a la carga de pareja que muestra 6 puntos porcentuales de diferencia entre el sexo masculino sobre el femenino.

\section{Relación de escolaridad y procedencia según el sexo de los trabajadores}

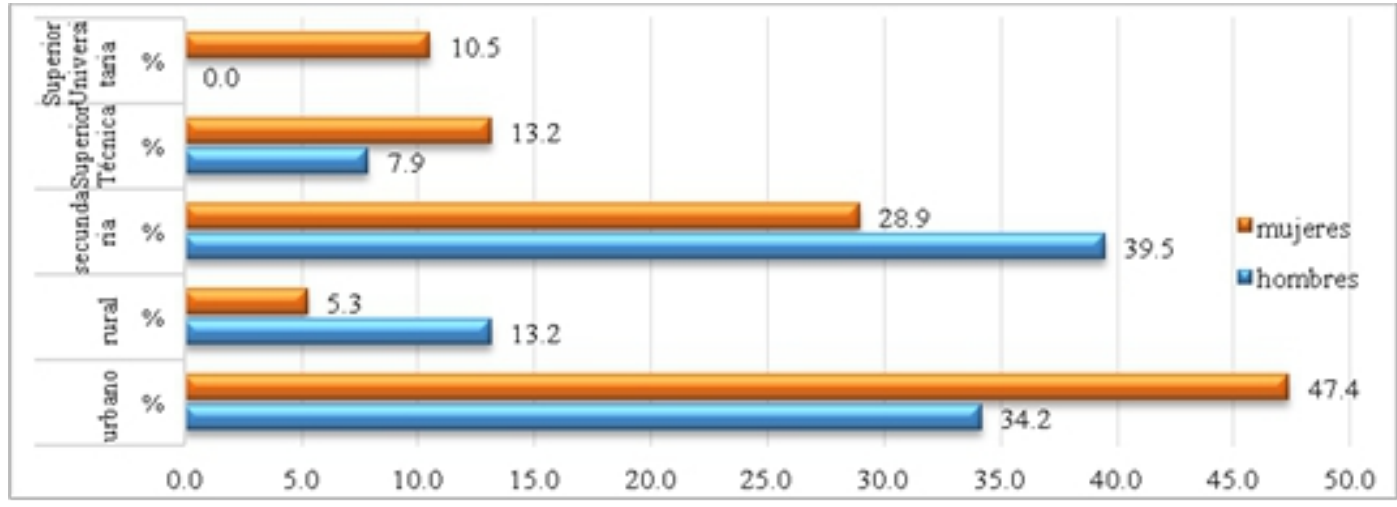

Gráfico 3. Relación de Escolaridad y Procedencia según sexo

Fuente: Investigación primaria, 2018.

Desde el lugar de procedencia se encuentra un predominio del sector urbano sobre el rural en ambos sexos, mayor presencia de las mujeres urbanas sobres los hombres urbanos con una diferencia de $13,2 \%$, y una presencia mayoritaria de hombres rurales sobre las mujeres rurales con una diferencia de 7,9\%. En cuanto al nivel de escolaridad se evidencia mejor preparación escolar del sexo femenino con referencia a su opuesto, 10,5\% de casos han llegado a cursar la educación superior universitaria, no observándose ningún caso para el sexo masculino, en la educación superior técnica, las mujeres predominan con una diferencia de 5,3\% frente a los hombres. Sin embargo, para cumplir con el requisito de acceder a la formación policial, el nivel de escolaridad como requisito básico es secundaria, y en esa categoría se 
encuentra que el $68,4 \%$ de los casos tienen este nivel de escolaridad, predominando el sexo masculino con el $39,5 \%$ frente al sexo femenino que presenta el 28,9\% de los casos. Asociando el estrés con el nivel de escolaridad y procedencia se concluye con la teoría de que las condiciones de vida si son factores influyentes en el desarrollo de la mayoría de morbilidades sociales (Giraldo, Toro, Macías, Valencia, \& Palacio, 2010).

\section{Relaciones entre las razones de selección del trabajo y la edad}

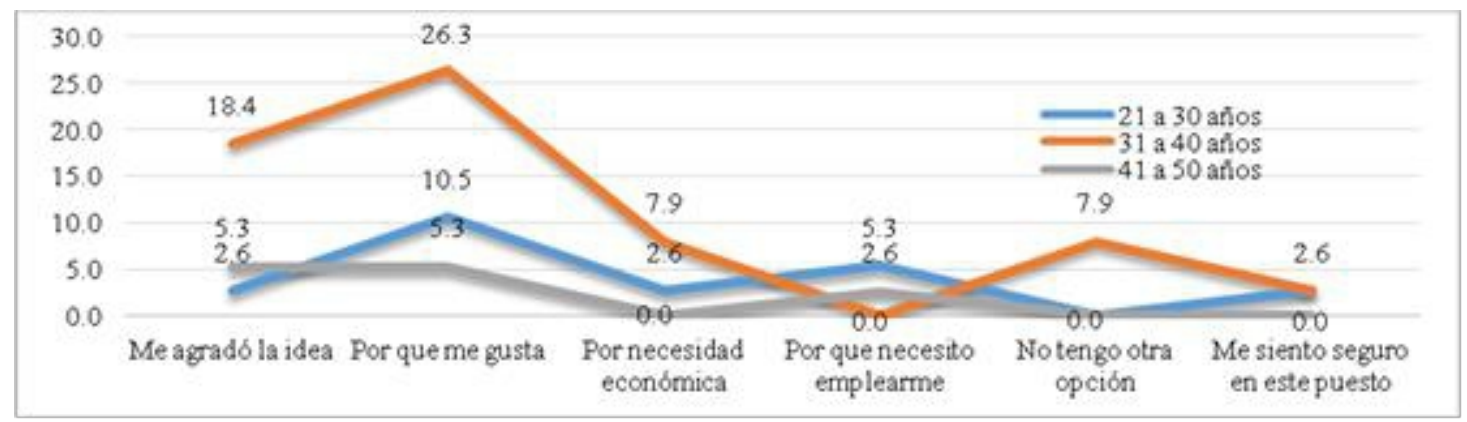

Gráfico 4. Principales razones de la selección del trabajo según edad Fuente: Investigación primaria, 2018.

Las razones por las que personas como el grupo objetivo, escogen sus diferentes actividades laborales es porque necesitan de un ingreso económico mensual, a veces ocurre que esas razones afectan la salud del trabajador, puesto que no fue escogido con algún anhelo o gusto personal, lo que genera inconformidades individuales, aumentando así las probabilidades de generar estrés o despersonalización. En el estudio, se determinan rangos de edad y razones por las que se escogió el trabajo de call center, la mayoría $(42,1 \%)$ escogió su trabajo porque le gusta, y porque le agradó la idea otro 26,3\%, alcanzando así un 68,4\% de trabajadores que desarrollan sus actividades a gusto porque les gusta, pero 3 de cada 10 trabajadores lo hacen por otras razones. El 10,5\% lo hace por la necesidad económica y se identifica como trabajadores que van de 21 a 40 años, otro grupo lo hace porque necesita emplearse, satisfacer una necesidad personal es una buena razón, pero a qué precio hablando de salud, un $8 \%$ lo hace porque no tiene otra opción, grupo que aumenta su posibilidad de generar estrés. 


\section{Sección 2: Diagnóstico de estrés- Síndrome Burnout} Agotamiento emocional por sexo y años de servicio

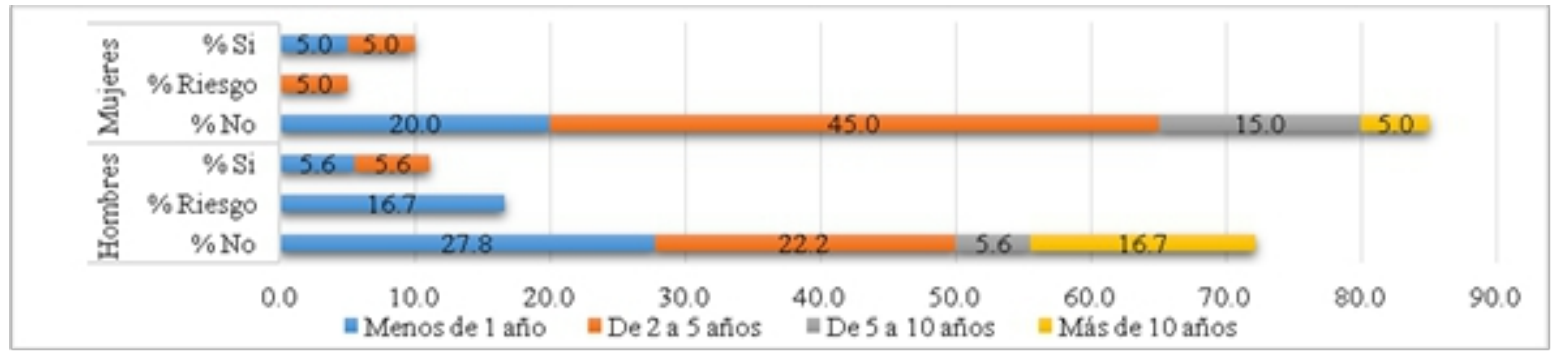

Gráfico 5 Agotamiento Emocional por sexo y años de servicio

Fuente: Investigación primaria, 2018.

Una parte del indicador del síndrome de Burnout es el Agotamiento Emocional, que está determinado según Maslach en su escala como, no presenta agotamiento, quienes no superan los 27 puntos medidos solamente de las 9 preguntas correspondiente al test (Facal, 2012), en el estudio se encontró que el 10,5\% del total de trabajadores tienen Agotamiento Emocional, y un porcentaje igual se encuentra en riesgo de padecer este trastorno. Esto se traduce a decir que 1 de cada 10 mujeres y uno de cada 10 hombres están padeciendo del síndrome de agotamiento emocional, igual relación se encuentra en riesgo para el caso de las mujeres (5\%) y son dos de cada 10 hombres quienes se encuentran en riesgo (16,7\%). El grupo que presenta el síndrome es el que tiene menor cantidad de tiempo en el desempeño de las labores como call center, lo cual indica que el estrés medido como agotamiento emocional obedece a la dinámica de acoplamiento en el sistema y en el tipo de actividades a desarrollar, situación que va decreciendo a medida que la persona va adquiriendo habilidades que bajan sus niveles de presión y angustia.

\section{Despersonalización por sexo y años de servicio}

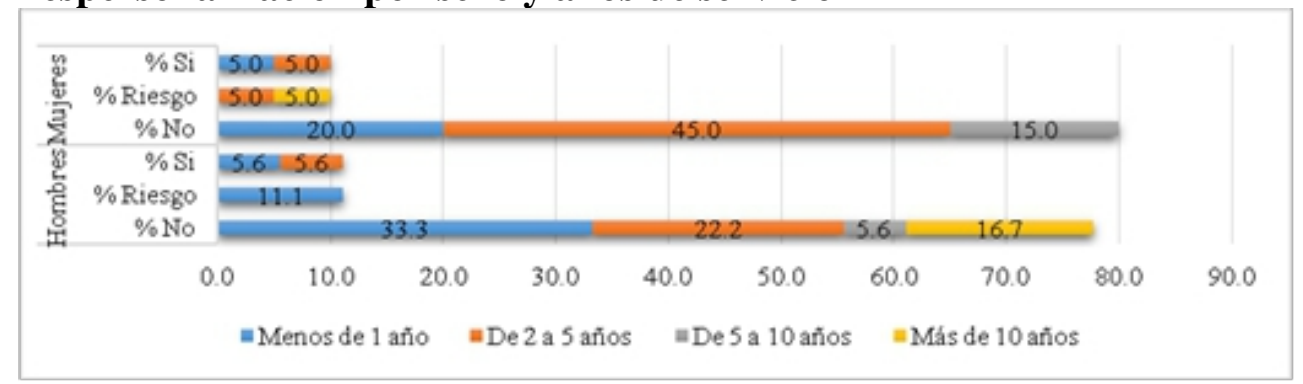

Gráfico 6. Despersonalización según sexo y años de servicio

Fuente: Investigación primaria, 2018.

El segundo componente que se mide en el proceso de diagnosticar Burnout es la despersonalización de los trabajadores, según la escala de 
Maslach este componente se evalúa sobre 30 puntos en 5 preguntas planteadas exclusivamente para medir este síndrome, los puntajes altos manifiestan la presencia del mismo, y los puntajes de 14 y menos excluyen la posibilidad de tenerlo (Pérez, 2010). En el estudio, se determina que existe un 10\% del sexo masculino que presenta esa despersonalización, y el 11,2\% para el caso femenino. Un porcentaje igual, se presenta en los trabajadores que se encuentran en riesgo de desarrollar este síndrome en ambos sexos. La relación que se desprende es reconocer que 1 de cada 10 mujeres y 1 de cada 10 hombres ha desarrollado este síndrome de despersonalización en el transcurso de su relación laboral, lo que es igual a decir que 2 de cada 10 trabajadores del call center tiene este padecimiento. El tiempo de relación laboral, al igual que el caso anterior del agotamiento emocional, es de quienes tienen menos de un año vinculados en el trabajo. Existe un 5\% de casos en que el grupo de trabajadores que tienen más de 10 años, se encuentra en riesgo o al límite de padecerlo.

\section{Realización personal por sexo y años de servicio}

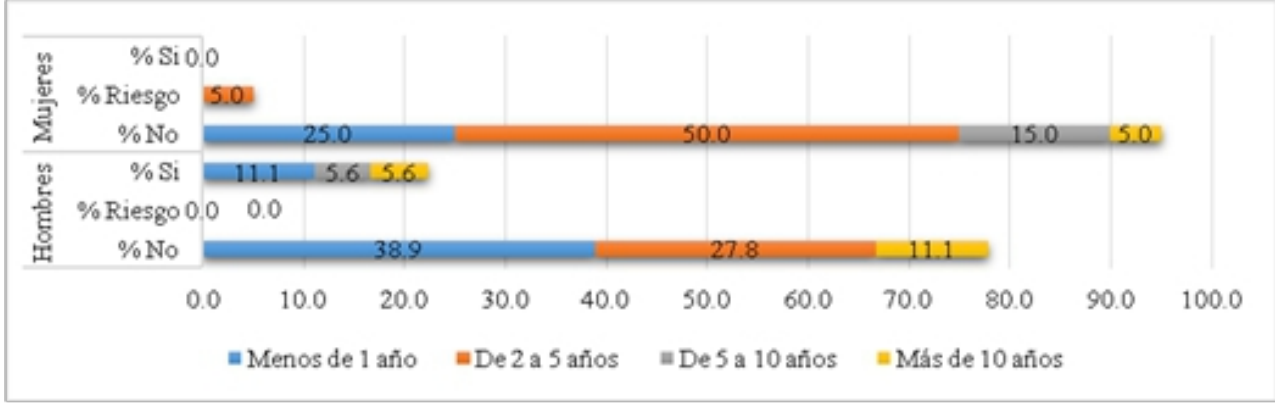

Gráfico 7. Realización personal según sexo y años de servicio.

Fuente: Investigación primaria, 2018.

El tercer componente que define el diagnóstico de Burnout es la realización personal, en este componente las puntuaciones son contrarias a las dos primeras, es decir, puntuaciones altas reflejan la realización de la persona por lo tanto es un resultado positivo que categoriza como no tener problemas de realización, más bien, puntuaciones por debajo de 17 indican problemas de estrés en la persona, por la no realización como tal (Facal, 2012). En los resultados se encuentra que no han podido realizarse en su lugar de trabajo el $22,3 \%$ y solo obedece al sexo masculino, lo cual demuestra que los hombres del grupo estudiado no cumplen acciones que les motive hacia la realización personal, influyendo así de manera negativa en su entorno individual de maneras diversas, otro hallazgo importante es encontrar que el 11,1\% tiene un tiempo de labores no mayor de un año, y el 11,2\% en cambio ocurren en quienes tienen más de 5 años de trabajo en la dependencia estudiada, pero se debe considerar que quienes conforman esta central son policías que han 
empezado así su carrera profesional, por lo que se puede prescindir que sea exclusivo por el rol de call center.

\section{Diagnóstico del Síndrome Burnout}

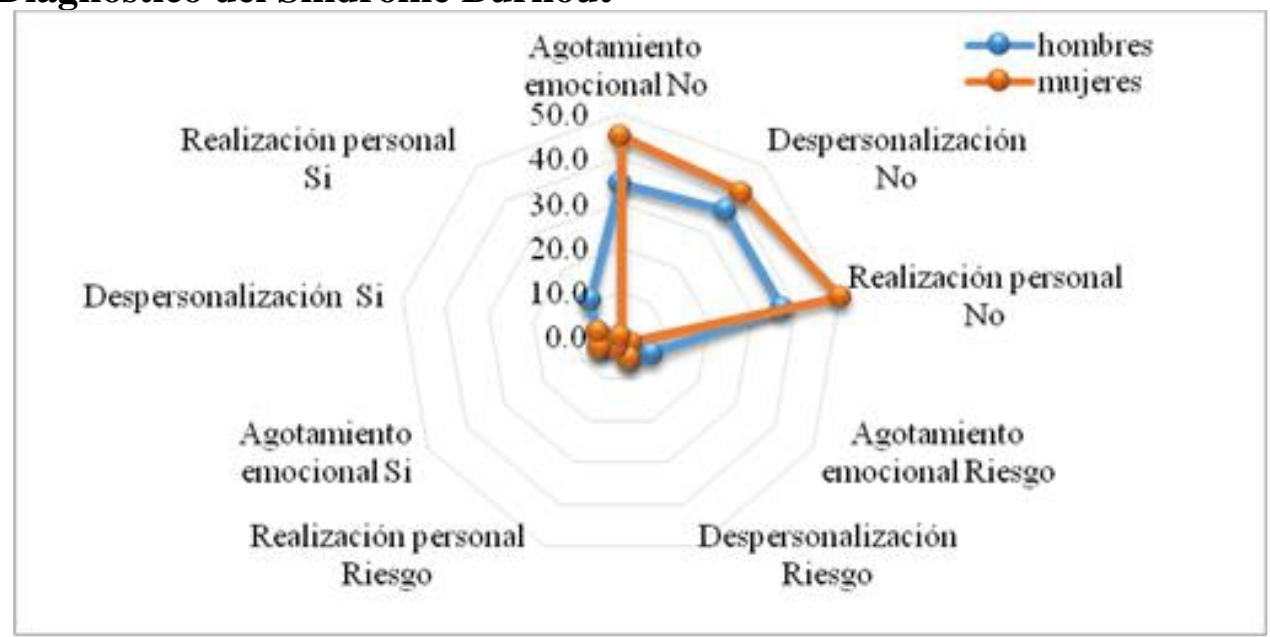

Gráfico 8. Diagnóstico del Síndrome Burnout

Fuente: Investigación primaria, 2018.

En la ilustración 8 se puede observar que existe una marcada tendencia hacia la zona del no, lo cual permite interpretar el test de Maslach que menciona, en relación al padecimiento del Burnout, que para que una persona presente el síndrome, debe tener en las dos primeras áreas: Agotamiento Emocional y Despersonalización, calificaciones o puntuaciones altas de acuerdo a las preguntas propias para su escala, y en la tercer área: Realización Personal, debe tener puntuaciones bajas, es decir debe cumplir con estas características para diagnosticar el síndrome, si en alguna de las categorías no se cumple esta relación, existe estrés pero no como se diagnostica Burnout (De Camargo, 2010). En el estudio, ningún trabajador cumple con estas tres condiciones, pero sí en alguna de las áreas de manera individual, por lo tanto, el grupo de trabajadores que son objetivo del presente documento no presentan el síndrome Burnout. Especial consideración debe mantenerse en los casos de estrés por despersonalización, agotamiento emocional y no realización personal, ya que cada uno es un factor de riesgo asociado que potencia el deterioro de la salud del trabajador. 


\section{Sección 3: Percepción del trabajador hacia el estado de su salud en general}

Percepción del estado de salud

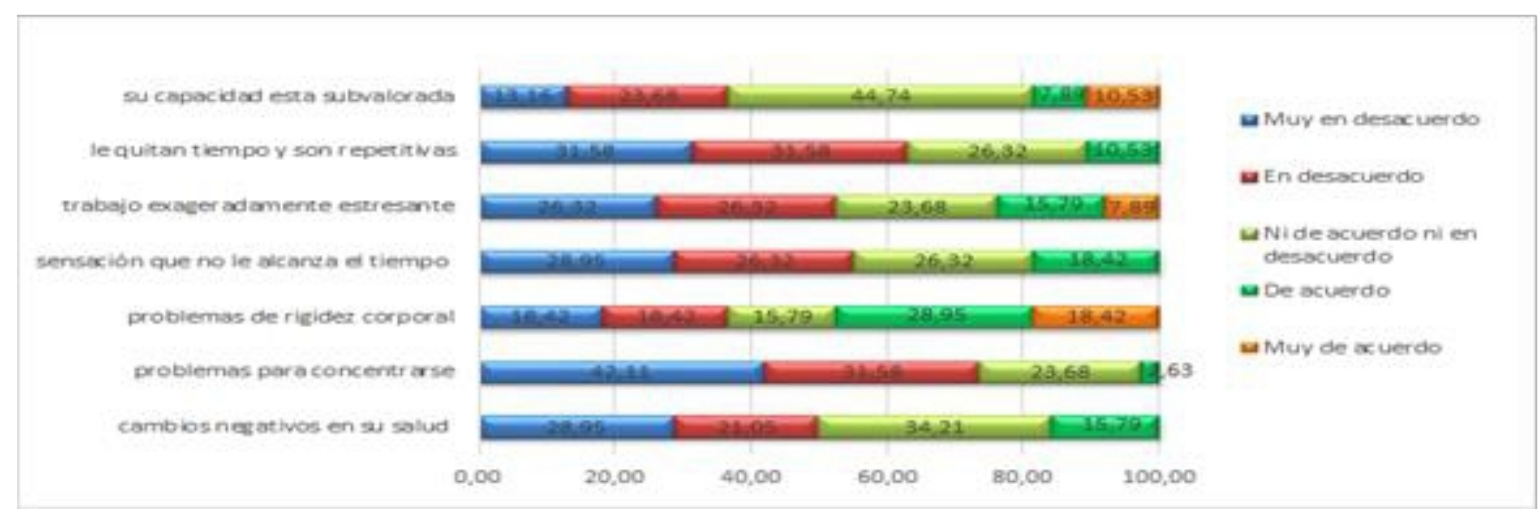

Gráfico 9. Percepción del estado de salud

Fuente: Investigación primaria, 2018.

La percepción es una situación subjetiva que depende exclusivamente de la persona y que, en el contexto de la investigación científica, precisa ser transformada en criterios objetivos que posibiliten la comprensión del fenómeno que se investiga (Bonilla \& Rodríguez, 2005). Para el estudio se considera todas las respuestas y se categoriza conforme escala planteada sin variar su contexto, las respuestas registradas tienen la tendencia hacia el no reconocimiento que existe un problema de salud, más del 72\% de trabajadores manifiestan estar en desacuerdo, y muy en desacuerdo de los enunciados propuestos. Existe solamente un reconocimiento en relación al problema de rigidez corporal, en donde el $47.37 \%$ reconoce y está de acuerdo que su salud se afectó por el contenido del postulado. En el análisis de promedios se concluye que el 15,8\% del total del grupo de hecho está muy en desacuerdo, el $34,2 \%$ en desacuerdo y el resto que es $50 \%$ están ni de acuerdo ni en desacuerdo, en el promedio global, los trabajadores no prevén alteraciones en su salud. 


\section{Sección 3.1: Percepción del trabajador respecto de sus Salud Física Percepción sobre la Afectación del Sistema Nervioso}

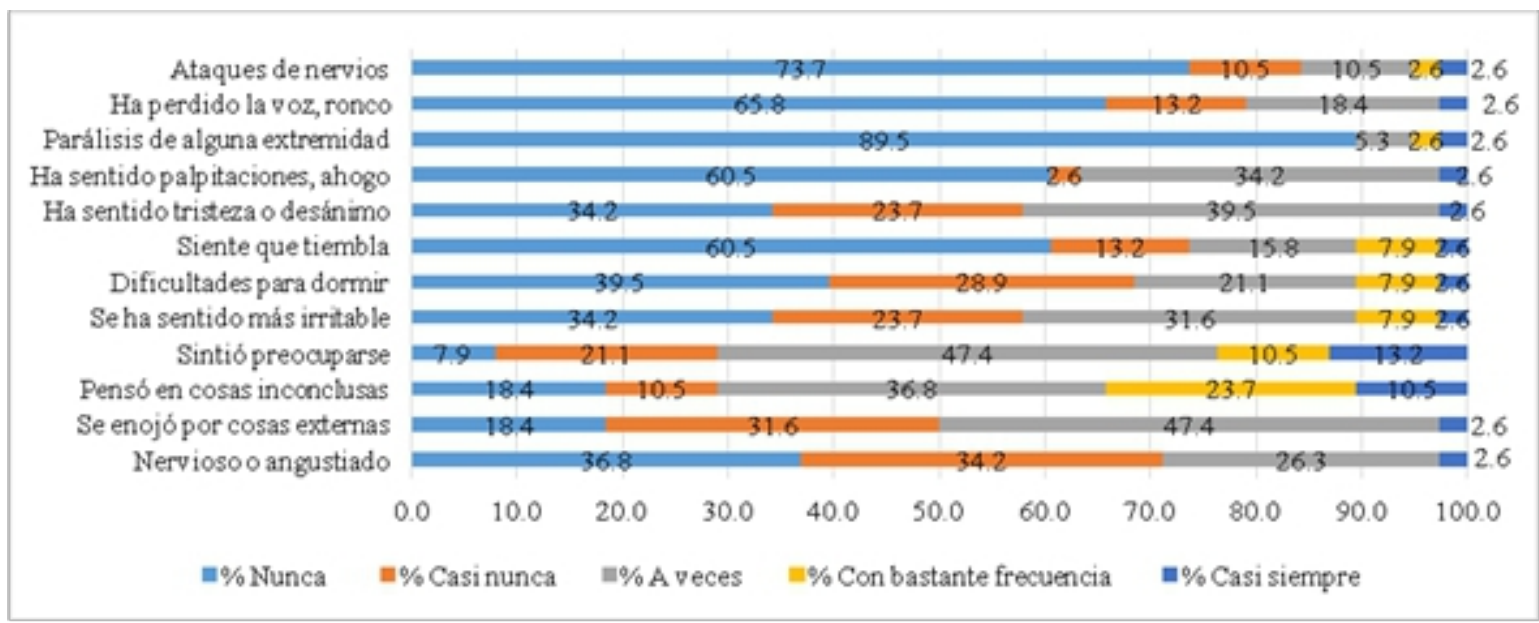

Gráfico 10. Percepción sobre la Afectación del Sistema Nervioso Fuente: Investigación primaria, 2018.

El estudio rescata cierta información, que está presente, pero en que en la globalidad del resultado se pierde, esto es el caso del 32,4\% de trabajadores que respondió las opciones de siempre y con bastante frecuencia, al referirse a la situación de permanecer pensando en las cosas que le quedaban por hacer, esta afectación origina problemas neuronales severos en cuanto a que generan preocupación de pensar en cosas que debe ejecutarse en el futuro, lo malo de este trastorno es que la persona por pensar en ese hecho, deja de vivir el presente y el pensamiento solo desea que pase el tiempo de manera rápida para correr a terminar las cosas que están pendientes, sin mencionar además que el estrés deja de ser individual y pasa a ser familiar y luego colectivo.

A este análisis se suma el $23,7 \%$ en el postulado que se pregunta si el trabajador sintió que tenía que preocuparse por ciertas situaciones laborales que están pendientes, lo cual corrobora que el trabajador prioriza su preocupación por las situaciones laborales y las pone sobre las preocupaciones familiares.

Otros síntomas identificados como problemas de la salud nerviosa es el sentirse irritable, no poder conciliar el sueño, y sentirse tembloroso, el estudio demuestra que estos síntomas lo han presentado el 10,5\% de total de trabajadores.

Estadísticamente, se generaliza a través de la razón que 1 de cada 10 trabajadores del call center presenta y reconoce como afectación de la salud nerviosa a la irritabilidad, la dificultad para dormir y el sentirse tembloroso.

Un pequeño porcentaje $(5,2 \%)$, sin dejar de ser importante, reconoce ya la presencia de ataques de nerviosismo luego de un evento que altera su 
tranquilidad, incluso en iguales porcentajes trabajadores que han sentido parálisis de alguna de sus extremidades luego de un evento similar.

Los resultados grupales, indican que el $15,79 \%$ de trabajadores manifiestan que nunca han dado muestras de afectación en su sistema nervioso, mientras que el 63,16\% manifiesta que casi nunca, por tanto, si se suman estas dos opciones el resultado sería del 78,95\%, en cambio otro grupo responde que a veces ha tenido manifestaciones, representan el 15,79\%, y en porcentajes iguales a $2,63 \%$ reconocen que con bastante frecuencia y siempre han tenido estas manifestaciones de afectación hacia su sistema nervioso, situación preocupante para la institución pues las acciones de salud ocupacional deben ser oportunas.

\section{Percepción sobre la Afectación del Sistema Digestivo}

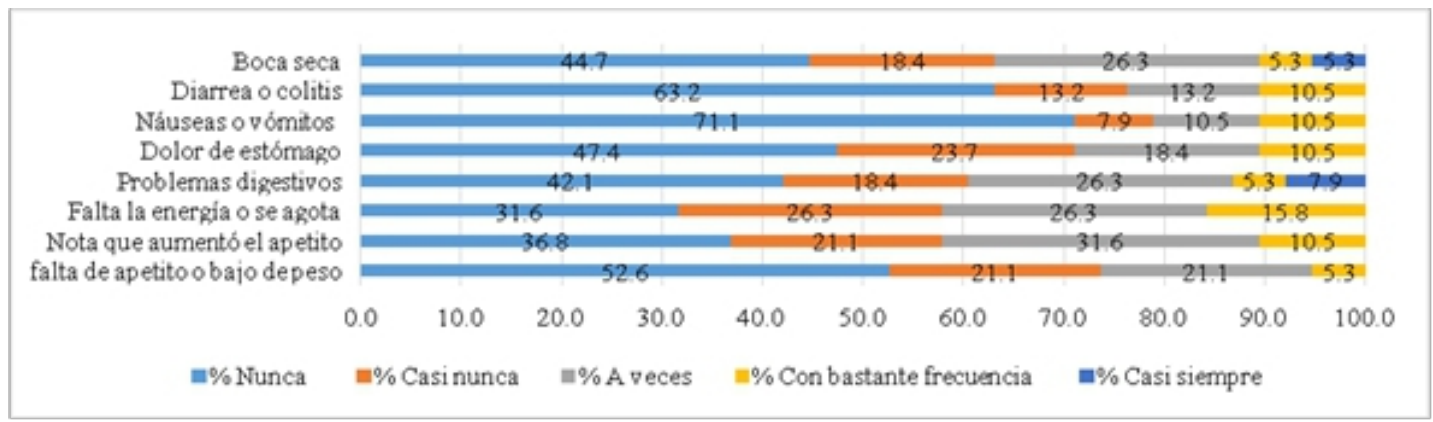

Gráfico 11. Percepción sobre la Afectación del Sistema Digestivo Fuente: Investigación primaria, 2018.

La investigación establece la necesidad de encontrar situaciones sintomáticas que orienten hacia un diagnóstico oportuno de entre las múltiples posibilidades que tienen los trabajadores de desarrollar una morbilidad. Al ser una imposibilidad, la ejecución con recursos médicos, laboratorio, imágenes y demás, se establece un banco de posibilidades o postulados que potencien la investigación desde la perspectiva del trabajador, aclarando que no se trata de una investigación clínica sino más bien una que trata de dimensionar la naturalización de los problemas de salud entre la sociedad.

Se pregunta sobre situaciones que pueden presentarse al momento de estar cursando una determinada morbilidad, haciendo uso de la frecuencia de los aparecimientos, las respuestas recogidas, muestran que la gran mayoría (89\%) de manera general responden que nunca, casi nunca y a veces han tenido alguna manifestación alterativa, aun cuando se pudo determinar que tienen algún determinado síntoma, el trabajador no lo acepta como un problema en su salud, y más bien maquilla la situación argumentando cualquier otra situación particular y a veces desligada.

La frecuencia de padecimientos indica la gravedad o agudeza del problema, lo cual es una muestra de que algo está surgiendo con rapidez en el 
interior del cuerpo, tal es así, que al preguntar si aumento o disminuyo el apetito en los últimos meses, las respuestas corresponden al $57,9 \%$ y $73.7 \%$ respectivamente a las respuestas de nunca y casi nunca, es decir, no se da importancia a la cantidad de alimento que ingiere ni a la necesidad alimentaria que se experimenta día a día.

Se calcula el promedio de las respuestas por cada ítem posible de selección, los resultados grupales, reflejan que el 28,94\% de trabajadores manifiestan que nunca han dado muestras de afectación en su sistema digestivo, mientras que el 55,26\% manifiesta que casi nunca, por tanto, si se suman estas dos opciones el resultado sería del $84,20 \%$, lo que es evidentemente la gran mayoría. Otro grupo responde que a veces ha tenido manifestaciones, representan el $10,53 \%$, y en porcentaje menor de 5,27\% reconoce que con bastante frecuencia han tenido estas manifestaciones de afectación hacia su sistema digestivo existen respuestas para la categoría de siempre.

\section{Percepción sobre la Afectación el Sistema Circulatorio}

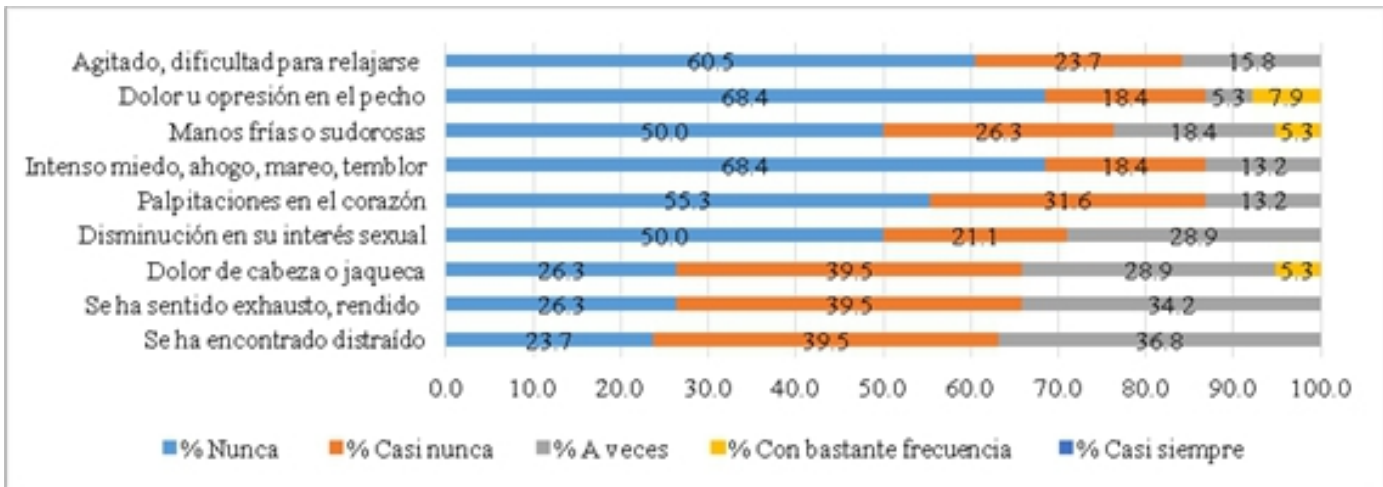

Gráfico 12. Precepción sobre la Afectación en el Sistema Circulatorio Fuente: Investigación primaria, 2018.

En este componente de evaluación, las respuestas en la gran mayoría obedecen a las opciones de nunca y casi nunca haber padecido alguna situación en particular que comprometa la afectación en el sistema circulatorio, esta gran mayoría en promedio suma $89,47 \%$ del total de respuestas recogidas de los trabajadores.

Nótese en la ilustración 12, que de las cinco opciones de respuesta, apenas y se visualizan tres, las dos primeras descritas anteriormente, y la tercera que obedece a la categoría de a veces representa el 10,53\%, en el cálculo global de los promedios, no aparece la opción "con bastante frecuencia”, no así en el descripción por proposición planteada donde aparece en tres: la primera con el $7,9 \%$ cuando se pregunta si ha tenido algún dolor $\mathrm{u}$ opresión en el pecho cuando ha tenido alguna alteración producto de una 
discusión o un mal rato, el 5,3\% igual en dos proposiciones: si ha sentido las manos frías o sudorosas y si ha tenido dolores de cabeza o cefalea al momento o después de una alteración o disgusto.

Para analizar con más detenimiento la gravedad del fenómeno que corresponde a esta descripción, se puede fijar en las respuestas de quienes marcaron como "a veces", luego se compara con las proposiciones y se encontró que el 34,2\% de trabajadores han identificado que existe un problema de agotamiento pues se han sentido exhaustos o rendidos, es decir, 3 de cada 10, estarían en riesgo de algún trastorno o padecimiento, o el caso del grupo de $28,9 \%$ que menciona haber tenido a veces dolores de cabeza o cefaleas, $\mathrm{y}$ otro con el mismo porcentaje que menciona haber disminuido su interés sexual, en hechos visibles se podrían especular diversos causales, pero al ser un dato discreto, se corre el riesgo de ignorarlo, corresponde entonces, a la salud ocupacional determinar el grado de afectación o no de los trabajadores en este componente de salud física y específicamente en el componente del sistema circulatorio (Aguilar, 2018).

Los siguientes resultados corresponden a la sección que es complementaria a esta, y tiene que ver con aspectos psicológicos del trabajador que están presentes e influyen en el estado de salud integral de la persona.

\section{Sección 3.2: Percepción del trabajador respecto al estado de sus Salud Psicológica \\ Percepción sobre la afectación en el Relacionamiento}

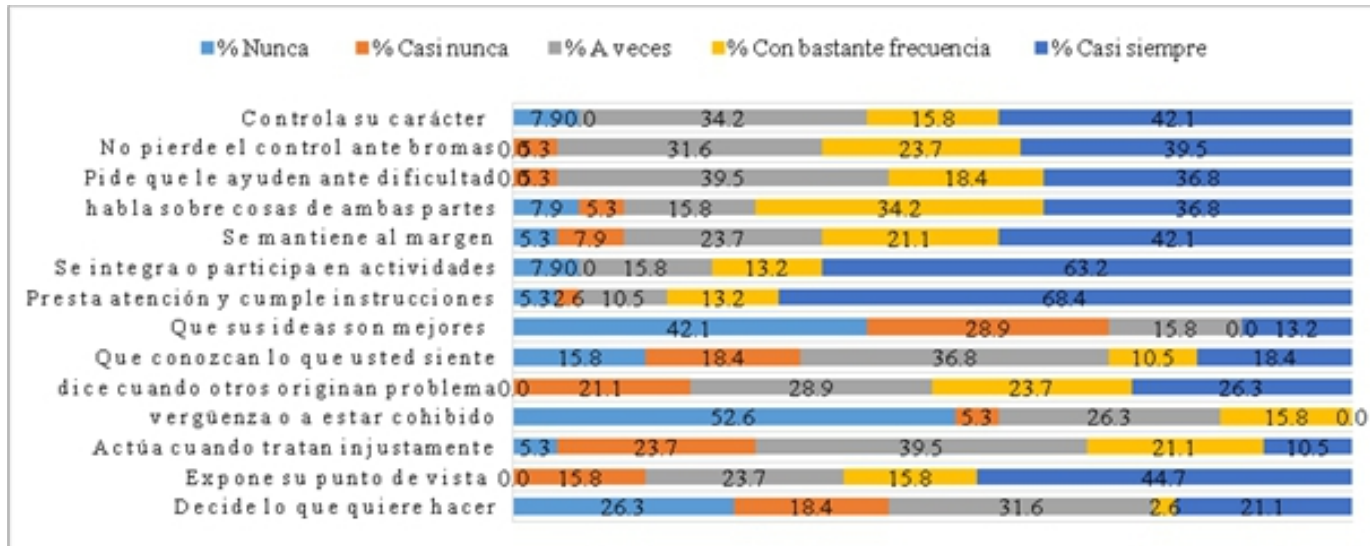

Gráfico 13. Percepción sobre la afectación en el Relacionamiento

Fuente: Investigación primaria, 2018.

Esta sección 3.2 corresponde al análisis de la situación psicológica que atraviesa el trabajador de un call center, es complementaria a la sección 3.1 que describió la afectación sobre la salud física del trabajador. 
El primer componente analizado es el de Relacionamiento, el cual analiza proposiciones de carácter, autocontrol, franqueza, ser frontal en sus decisiones, mantenerse al margen de las discusiones, integración en las actividades, vergüenza, entre otras (Piqueras Rodríguez, Ramos Linares, Martínez González, \& Oblitas Guadalupe, 2009).

Dentro de la propia escala de medición, la directriz del autor de la misma manifiesta que puntajes de 35 a 67 puntos indican un buen relacionamiento, es decir, la escala mide en la globalidad y en la forma como se interactúa en el contexto de las proposiciones, de 28 a 34 puntos obtenidos de igual manera en la globalidad del test significa que la persona tiene problemas de relacionamiento, y puntajes con $27 \mathrm{y}$ menos puntos indican que la persona tiene un mal relacionamiento.

Con este detalle de análisis, el resultado global muestra que tan solo el $2,63 \%$ del personal tiene mal relacionamiento y el $97,37 \%$ tiene buen relacionamiento. Con estas estadísticas se extrapola el propósito de la investigación de decir que el relacionamiento con problemas o el mal relacionamiento ocasionan un mal funcionamiento en la estructura y dinámica del trabajo, por lo que se demuestra que en el caso del grupo de estudio este problema no está presente.

Sin quitar importancia al uso o a la aplicación del instrumento, se debe recoger algunos datos estadísticos que pueden ser el insumo para establecer la propuesta de solución, como el caso del $42,1 \%$ que describe que nunca y casi nunca controla su carácter ante determinadas situaciones, el 15,8\% a veces si controla y otras no, es decir, presumiblemente existen ciertos parámetros que pueden estar afectando la salud psicológica sin ser notorio en la globalidad del análisis.

\section{Afectación en la autoestima}

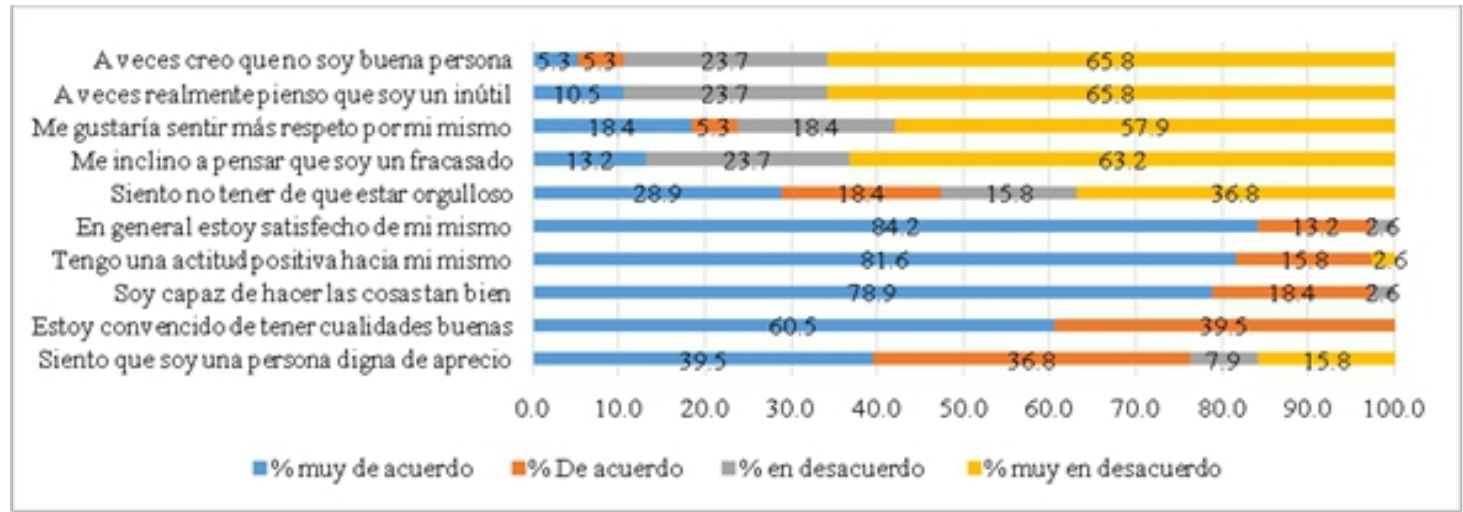

Gráfico 14. Afectación en la autoestima

Fuente: Investigación primaria, 2018. 
El test empleado mide el nivel de autoestima a través del análisis de varias proposiciones planteadas a la persona, para poder hacerlo, se expresan 10 proposiciones de entre las cuales el $50 \%$ son planteadas de manera positiva y se puntúan de 4 a 1 mientras que el otro $50 \%$ son planteadas de manera negativa y se puntúan al inverso, es decir de 1 a 4 , la suma de todas las respuestas a las proposiciones, el autor plantea una escala de valoración que va de 3040 puntos, la persona tiene autoestima elevada, de 26 a 29 puntos autoestima media y menos de 25 puntos autoestima baja.

Los resultados acordes a esta aplicación correcta de la escala, se establece que en el grupo solo se presentan dos tipos de autoestima solamente, el $52,63 \%$ de los trabajadores tiene una autoestima media y el $47,37 \%$ una autoestima baja. Este mismo resultado medido por el sexo del trabajador se establece de quienes tienen autoestima media, el 28,95\% son hombres, el $23,68 \%$ son mujeres, esto sobre el total del grupo investigado, y de quienes tienen la autoestima baja corresponden al 18,42\% de hombres y el 28,95\% de mujeres.

Existe una tendencia de un grupo de trabajadores que manifiesta estar en desacuerdo cuando se pregunta si es un persona digna de aprecio $(23,7 \%)$ lo cual ratifica que existe una situación no acorde a un razonamiento más elaborado, pero el fenómeno se repite bordeando el $50 \%$ de quienes lo aseguran, y es que el 10,6\% responde estar de acuerdo cuando se pregunta si cree que no es una buena persona, lo que se quiere reflejar es, que existe un grupo de trabajadores que en la globalidad se pierden, lo cual hace pensar que es necesario una intervención objetiva de salud ocupacional en este conglomerado, pues el hallazgo motiva a descubrir de manera individual quienes son los trabajadores que necesitan ayuda para mejorar su condición de estima hacia sí mismo.

\section{Afectación en la Asertividad}

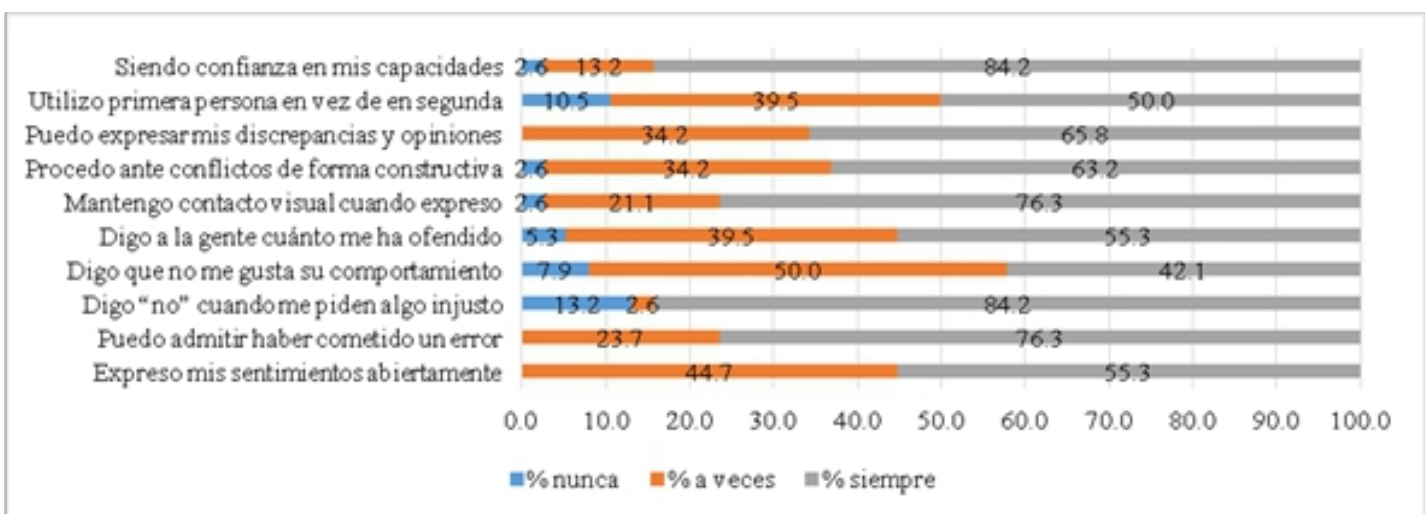

Gráfico 15. Afectación en la Asertividad

Fuente: Investigación primaria, 2018. 
Al ser una situación igual a las dos anteriores, la herramienta que se utilizó es un test que mide desde un conjunto de proposiciones, el grado de asertividad o no de una persona, lo cual reflejaría su estado de situación dentro de un grupo social. El test mide dificultad de ser asertivo con una escala de puntuación de menos a 16 puntos, ligeramente asertivo con puntuaciones de 17 a 21, normalmente asertivo con puntuaciones de 22 a 26, y asertivo de forma constante o en la mayoría de las situaciones a quienes puntúen entre 27 y 30 puntos.

El resultado global de la aplicación del test demuestra que el 2,63\% de trabajadores tiene dificultad de ser asertivo, lo cual por demás se explicaría como un factor que influye en el relacionamiento con los demás, por tanto, existe un grupo pequeño de trabajadores que tienen sintomatología acorde a la urgencia y necesidad de intervención con salud ocupacional. Existe otro grupo que representa el 5,26\% del global de trabajadores que se categorizan como "ligeramente asertivos", es decir, no tienen una capacidad definida para reaccionar ante situaciones de relacionamiento, en el ámbito laboral, esto es una muestra o foco de preocupación por las potenciales posibilidades de tener reacciones no asertivas por parte del trabajador que profundizaría un problema de nivel humano.

Los categorizados como "normalmente asertivos" corresponden al $36,84 \%$ del total de trabajadores, lo que presta espacial tranquilidad ante situaciones que pueden generarse desde el lado contrario a este tipo de comportamiento, pero que no deja de ser digno de observación, y por fortuna, más de la mitad de trabajadores son "asertivos de forma constante", es decir, el 55,26\% del total de trabajadores tendrán reacciones positivas de asertividad ante una determinada situación, lo cual ayuda a aplacar los problemas de asertividad de la minoría del grupo.

\section{Sección 4: Percepción del trabajador respecto a su rendimiento laboral Percepción con respecto a la ejecución de actividades}

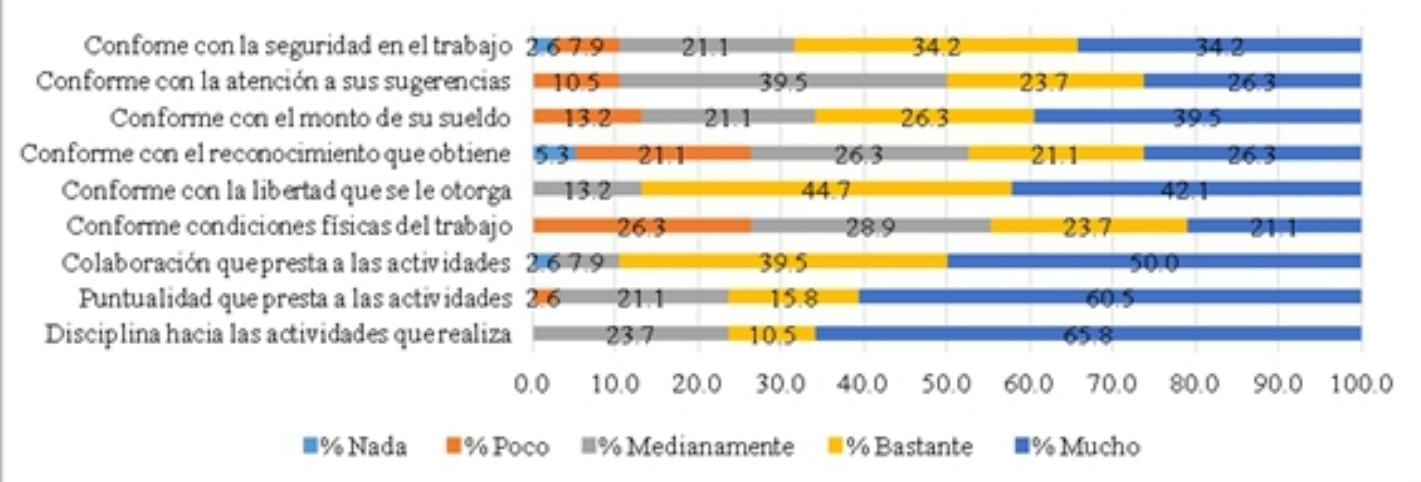

Gráfico 16. Percepción con respecto a la ejecución de actividades.

Fuente: Investigación primaria, 2018. 
Este componente mide la percepción de los trabajadores con respecto a situaciones que tienen que ver con la ejecución de actividades en su lugar de trabajo, las proposiciones empleadas para medir tienen que ver con la seguridad en el trabajo, la estabilidad, los ingresos, el reconocimiento que obtiene, la libertad, las condiciones físicas, la colaboración, puntualidad y disciplina hacia las actividades que debe desarrollar (Salas Perea, Díaz Hernández, \& Pérez Hoz, 2013).

Estas proposiciones se miden en la globalidad de los hechos, usando escalas de mucho, bastante, medianamente, poco y nada, haciendo énfasis a la ejecución de actividades como tal. Los resultados globales que son calculados de promediar las respuestas de cada proposición y categorizadas acorde a la escala de medición, se obtuvo que el $28,95 \%$ manifiesta que ejecuta "mucho" de sus actividades, el 47,37\% ejecuta "bastante" de sus actividades, el $21,05 \%$ ejecuta "medianamente" sus actividades, y el 2,63\% ejecuta "poco" de sus actividades.

En este componente si se puede analizar de manera individual cada proposición, como el caso que menciona si está conforme con el sueldo que gana, las respuestas esperadas suponen un grado de conformidad de bastante conforme o muy conforme, pero tan solo el 65,8\% de ellos, lo manifiesta así, contrariamente al $21,1 \%$ que su conformidad es medianamente, y el 13,2\% tiene poca conformidad. En suma 3 de cada 10 trabajadores tienen dificultades de aceptación y conformidad con su salario, entonces, existirá una buena calidad en el desempeño de las actividades que realizan, sería el motivo de una nueva función de la salud ocupacional. En cuanto a la disciplina que ponen los trabajadores a sus actividades que realizan se encuentra que el 76,3\% de ellos, manifiesta como bastante y mucha disciplina, contrariamente al 23,7\% que manifiesta que aplica medianamente la disciplina hacia las actividades que realiza.

\section{Percepción con respecto al logro de objetivos}

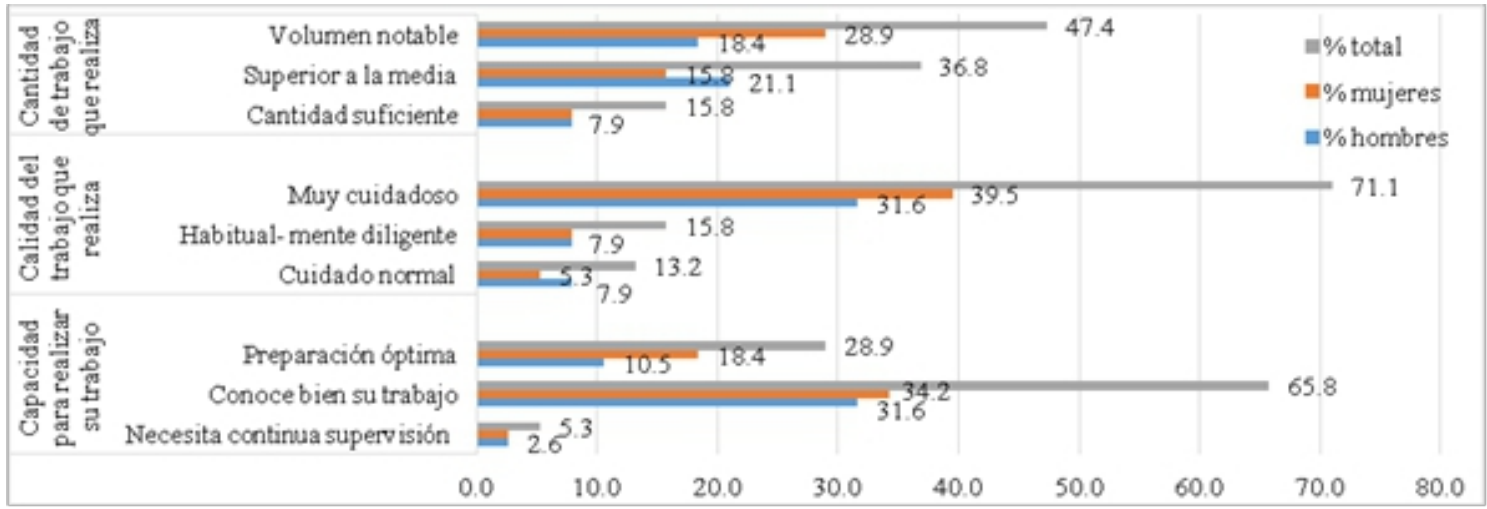

Gráfico 17. Percepción con respecto al logro de objetivos

Fuente: Investigación primaria, 2018. 
En este componente, la investigación propone entender si desde la percepción del propio trabajador de una empresa o institución se valora su propio esfuerzo y el esfuerzo colectivo para la consecución de los objetivos institucionales, puesto que muy a menudo, las individualidades sugieren empujar objetivos individuales, se desconocen los objetivos institucionales, por lo tanto; se hacen esfuerzos innecesarios y hasta exigentes por no tener esta claridad (López-Trejo, et al., 2018).

Se valora entonces, la cantidad de trabajo que realiza, el 47,4\% de trabajadores dice que lo hace en volumen notable, otro grupo que corresponde al 36,8\% manifiesta que su producción es superior a la media y el 15,8\% trabaja para la cantidad suficiente. En cuanto al pensamiento de producción según género, el femenino $(28,4 \%)$ afirma que el volumen de producción es notable frente al $18,4 \%$ de su opuesto, de quienes afirmaron que trabajan superior a la media se diferencia los hombres con el $21,1 \%$ superior a las mujeres que representan el 15,8\%, mientras que existe una igualdad entre géneros $(7,9 \%)$ de quienes trabajan en cantidad suficiente. No existen respuestas que indiquen producciones de insuficientes o escasas, que fueron también investigadas y propuestas como alternativas.

En cuanto a la Calidad del trabajo que realiza el $71,1 \%$ del total de trabajadores manifiesta que es muy cuidadosa en cuanto al trabajo que realiza, de ellos el 39,5\% corresponden a las mujeres que superan por muy poco a los hombres que representan el 31,6\%, de manera general ese es un buen resultado de una institución que labora en servicio, puesto que cuenta con el apoyo y la dedicación de sus miembros. Otro porcentaje indica que en cuanto a calidad de su trabajo es habitualmente diligente, representa a este grupo el 15,8\% que se reparten exactamente por igual entre los dos géneros, y el 13,2\% del total menciona que su calidad tiene un cuidado normal. En este componente tampoco se obtuvo respuestas de "errores frecuentes" y de "errores excesivos" que también estaban propuestos.

En cuanto a la Capacidad para realizar el trabajo, se midieron situaciones de sentirse en la capacidad plena de desarrollar sus determinadas actividades laborales, considerando la opción de "preparación óptima" con un total de respuestas que corresponden al 28,9\% divididas en el 18,4\% de mujeres que opinaron frente al $10,5 \%$ de hombres, la mayoría de los trabajadores analiza este componente como "conozco bien mi trabajo" correspondiendo al $65,8 \%$, divididos entre el 34,2\% para mujeres y el $31,6 \%$ para hombres. No se encuentran diferencias en cuanto al sexo de los trabajadores y sus tendencias hacia la capacidad.

Por último, el grupo que respondió la opción de "necesita continua supervisión" representa el 5,3\% del total de trabajadores compartiendo de manera igual los porcentajes correspondientes del 2,6\% los dos sexos. 


\section{Rendimiento laboral desde la Satisfacción Individual}

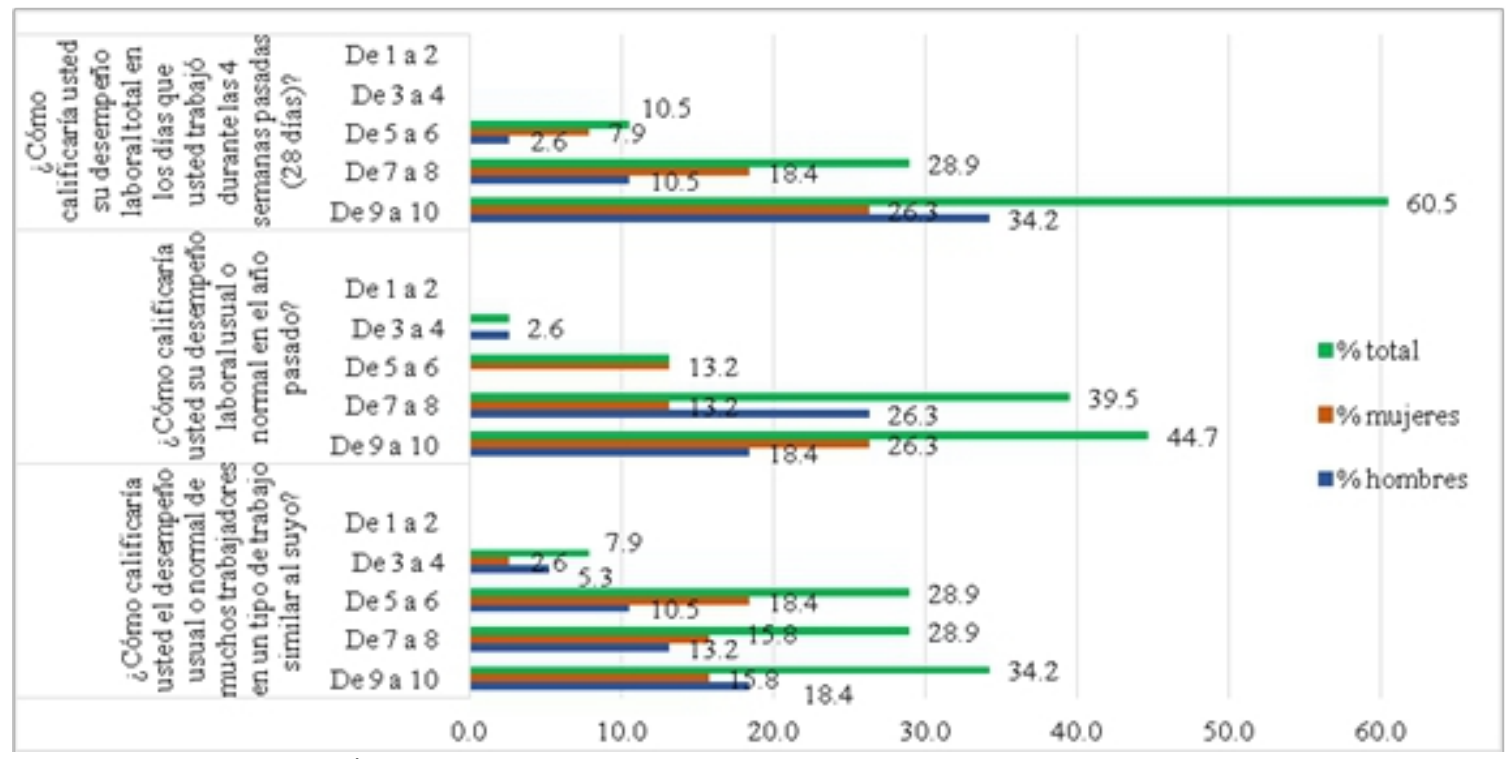

Gráfico 18. Rendimiento laboral desde la satisfacción individual

Fuente: Investigación primaria, 2018.

La autoevaluación sobre el rendimiento laboral es uno de los componentes finales que planteó la investigación, en principio se plantea que sean los trabajadores quienes valoren e interpreten su situación de efectividad en el trabajo. Los resultados aportan en gran medida en la toma de decisión de quienes son responsables de velar por la calidad del talento humano que exige una determinada institución a sabiendas que el capital humano es el valor más importante en la producción de servicios de una empresa.

A la proposición de calificar el desempeño usual o normal de muchos trabajadores en un tipo de trabajo similar al de quien responde, las respuestas apuntan en varias direcciones y son dadas bajo la percepción individual, es decir, todos califican lo del resto, bajo esta aclaración el promedio final de la valoración grupal para esta proposición es de 7,34 puntos sobre 10 que fue la calificación mayor.

En el rango de evaluación institucional que maneja una medida por competencias, este valor grupal sería igual a decir que el promedio del desempeño es de 3,67/5 lo que representa que alcanza los objetivos, llena las expectativas. En este rango del promedio, el grupo respondió en un porcentaje total de $28,9 \%$, igual valor quienes calificaron de 5 a 6 puntos, y los que dieron mejor calificación, de 9 a 10 fueron el 34,2\%. Llama la atención quienes dieron puntuaciones entre 3 y 4 que son el 7,9\% del total del grupo, las razones pueden ser varias, pero no fueron planteadas por la investigación. 
En cuanto a la calificación del desempeño personal, se solicitó que la gente se autoevaluara en dos momentos de tiempo, la primera evaluación, que sea un análisis del desempeño realizado en el último año de labores, y la segunda en un análisis del desempeño desarrollado en el último mes de labores, en estas dos situaciones el trabajador tiene la oportunidad de analizarse, reflexionar y globalizar determinadas acciones que suman hasta formar un todo, lógicamente que los resultados deberían ser iguales en los dos ámbitos de tiempo, puesto que la calidad y el rendimiento debe ser igual todos los días de labores (Pedraza, Amaya, \& Conde, 2010).

Los resultados fueron del 44,7\% con calificaciones de 9 a 10 puntos, notas máximas del rendimiento en la escala propuesta, de esto, el 26,3\% corresponde a la autoevaluación de las mujeres, frente al 18,4\% de calificaciones realizadas por hombres. El grupo que calificó su rendimiento con 7 a 8 puntos es el 39,5\%, en donde son los hombres quienes tienen mayor presencia con el $26,3 \%$ frente al femenino que corresponde al 13,2\%. El promedio de evaluación global del grupo en este componente es de 8 puntos, lo cual se traduce en un buen desempeño, autodeterminado desde la satisfacción individual.

El tercer parámetro evaluado, como se describió, es la autovaloración del desempeño en el período del último mes de labores, las respuestas fueron sumamente altas y muy diferentes al comportamiento de las dos primeras, el $60,5 \%$ califico su desempeño con valores de 9 a 10 puntos, de los cuales, el $34,2 \%$ son hombres y el $26,3 \%$ son mujeres, en este hallazgo no se comprende ciertamente por qué difieren los resultados según sexo, acaso son otros los pensamientos a la hora de responder con la verdad y a la vez al momento de reflexionar sobre lo que se está haciendo.

\section{Conclusiones}

Mediante las encuestas aplicadas, se demostró la existencia de estrés laboral en el grupo investigado, pero no la presencia del síndrome de Burnout, sin embargo, existen evidencias de afectaciones por agotamiento emocional en un $10,5 \%$, despersonalización $22,2 \%$ y baja realización personal $22,3 \%$, sin contar el grupo que se encuentra en riesgo de padecerla. Las condiciones laborales ayudan a que no exista el síndrome porque es un grupo que realiza actividad física frecuente.

Se identifican las afectaciones físico-psicológicas asociadas al estrés, las más frecuentes provienen de problemas en la salud física, específicamente en el sistema nervioso ( 1 de cada 10) y en menor proporción en el digestivo $15,8 \%$, no aparecen problemas en el sistema circulatorio, por lo tanto, existe afectación en el rendimiento laboral, aunque para el trabajador éste no los asocia a los problemas de salud física. Concluyendo que los resultados son mayoritarios porcentualmente aislados debido a que el grupo de estudio realiza 
frecuente actividad física, lo que disminuye considerablemente los efectos secundarios y negativos en su salud física.

\section{References:}

1. Aguilar, M. (2018). El estrés y su influencia en la calidad de vida. MULTIMED Revista Médica Granma, 21(6).

2. Bonilla, E., \& Rodríguez, P. (2005). Más allá del dilema de los métodos; La investigación en las Ciencias Sociales (Tercera ed.). Bogotá: Norma. Recuperado el 25 de mayo de 2018, de https://books.google.es/books?hl=es\&lr=\&id=REOIWoQuAL4C\&oi $=$ fnd $\&$ pg $=$ PA $17 \& d q=\mathrm{La}+$ percepci\%C3\%B3n+es+una+situaci $\% \mathrm{C} 3 \%$ $\mathrm{B} 3 \mathrm{n}+$ subjetiva+que+depende+exclusivamente+de+la+persona+y+qu e,+en+el+contexto+de+la+investigaci\%C3\%B3n+cient $\%$ C3\%ADfica ,+ precisa+ser+transformada+

3. De Camargo, B. (2010). Estrés, síndrome general de adaptación o reacción general de alarma. Revista Médico Científica, 2(17), 78-86. Recuperado el 21 de mayo de 2018

4. Facal, T. (2012). Prevalencia del Síndrome de Burnout en trabajadores sociales de los servicios sociales comunitarios. Portularia, 12(1), 5969. Recuperado el 20 de mayo de 2018, de http://www.redalyc.org/html/1610/161024645007/

5. Giraldo, A., Toro, M. Y., Macías, A. M., Valencia, C. A., \& Palacio, S. (2010). La promoción de la salud como estrategia para el fomento de estilos saludables. Hacia la Promoción de la Salud, 15(1), 128-143. Recuperado el 20 de mayo de 2018, de http://www.scielo.org.co/scielo.php?script=sci_abstract\&pid=S012175772010000100010

6. Houtman, I., Jettinghoff, K., \& Cedillo, L. (2008). Sensibilizando sobre el Estrés Laboral en los Países en Desarrollo. Francia: Organización Mundial de la Salud.

7. Jiménez, B. M., \& León, C. B. (2010). Factores y riesgos psicosociales, formas, consecuencias, medidas y buenas prácticas. Madrid: Universidad Autónoma de Madrid.

8. Juszczyk, P., Paraschiv, G., \& Szymanska, A. (2009). Juszczyk, P., Paraschiv, G., Szymanska, A., Kolodziejczyk, A. S., RodziewiczMotBinding epitopes and interaction structure of the neuroprotective protease inhibitor cystatin $C$ with $\beta$-amyloid .

9. López-Trejo, H., Carrera-Salazar, C., Eurrieta-Ortíz, M., GarcíaArroyo, L., Gómez-Márquez, M., Llanillo-Navales, J., . . . GonzálezPérez, M. (2018). Análisis del Estrés Laboral y su Repercución en la Salud Física y Mental en Operadores de Tracto Camión. European Scientific Journal, 14(11), 10-22. doi:10.19044/esj.2018.v14n11p10 
10. Meza, A., \& Lazarte, C. (1984). Asertividad: problemas de definición y de medición-versión preliminar de una prueba. Revista de Psicología, 2(1), 5-31.

11. Moriana Elvira, J. A., \& Herruzco, J. (2005). El síndrome de burnout como predictor de bajas laborales de tipo psiquiátrico. Clínica y Salud.

12. Motowidlo, S. (2004). Job performance. Handbook of Psychology: Industrial and Organizational Psychology, 39-53.

13. Pedraza, E., Amaya, G., \& Conde, M. (2010). Desempeño laboral y estabilidad del personal administrativo contratado de la Facultad de Medicina de la Universidad del Zulia. Pedraza, E., Amaya, G., \& Conde, M. (2010). Desempeño laboral y estabilidad del personal administRevista de Ciencias Sociales, 16(3), 493-505.

14. Pérez, A. M. (2010). El sindrome Burnout, evolución conceptual y estado actual de la cuestión. Vivar Academia, 112, 42-80. Recuperado el 20 de mayo de 2018, de http://www.vivatacademia.net/index.php/vivat/article/view/192

15. Piqueras Rodríguez, J. A., Ramos Linares, V., Martínez González, A. E., \& Oblitas Guadalupe, L. A. (2009). Emociones negativas y su impacto en la salud mental y física. Suma psicológica.

16. Restrepo, H. E., \& Málaga, H. (2011). Promoción de la salud: cómo construir vida saludable. Pan American Health Org.

17. Roldán, E. (2004). Género, políticas locales e intervención social. Madrid: Editorial Complutense.

18. Salas Perea, R. S., Díaz Hernández, L., \& Pérez Hoz, G. (2013). Identificación y diseño de las competencias laborales en el Sistema Nacional de Salud. Educación Médica Superior, 27(1), 92-102.

19. Segurado Torres, A., \& Agulló Tomás, E. (2004). Calidad de vida laboral: hacia un enfoque integrador desde la Psicología Social. Psicothema:

20. Segurado Torres, A., \& Agulló Tomás, E. (2002). Calidad de vida laboral: hacia un enfoque integrador desde la Psicología Social. Psicothema, 14(4).

21. Silla, J. M. (2011). El estrés laboral: una perspectiva individual y colectiva. Prevención, trabajo y salud. Revista del Instituto Nacional de Seguridad e Higiene en el trabajo, , 13, $(18,38)$. 